DOI:10.2478/rrlm-2021-0002

\title{
Evaluation of Plasma AA/DHA+EPA Ratio in Obese Romanian Children
}

\author{
Adina Huțanu ${ }^{1,2^{*}}$, Mihaela Zaharia ${ }^{1,2}$, Lenard Farczadi ${ }^{2}$, Ionela Maria Paşcanu ${ }^{3}$, \\ Raluca Monica Pop ${ }^{3,4}$, Minodora Dobreanu ${ }^{1,2}$
}
1. Department of Laboratory Medicine, George Emil Palade University of Medicine, Pharmacy, Science, and Technology of Targu Mures, Romania

2. Center for Advanced Medical and Pharmaceutical Research, Laboratory of Humoral Immunology, George Emil Palade University of Medicine, Pharmacy, Science, and Technology of Targu Mures, Romania

3. Endocrinology Department, George Emil Palade University of Medicine, Pharmacy, Science, and Technology of Targu Mures, Romania

4. Research Methodology Department, George Emil Palade University of Medicine, Pharmacy, Science, and Technology of Targu Mures, Romania

\begin{abstract}
The aim of the study was to evaluate the plasma profile of arachidonic acid (AA), docosahexaenoic acid (DHA), and eicosapentaenoic acid (EPA), as well to analyze the relationship of Omega 6/Omega 3 ratio with anthropometric parameters and insulin resistance markers. Material and methods: Plasma levels of free fatty acids (FFAs) were measured using a high-throughput LC-MS AB Sciex4600 in 202 children (127 obese and 75 non-obese), age and sex-matched. Lipid and glucose profiles were assessed with current laboratory methods, while insulin resistance and beta-cell function were evaluated using HOMA-IR and HOMA- $\beta$ respectively. Results: In obese children, $A A$ and $A A /(D H A+E P A)$ ratio were significantly higher regardless of age and gender. In the lowest quartile of $D H A$, there was a clear trend for insulin resistance, with plasma insulin level, HOMA-IR, and HOMA- $\beta$ significantly higher compared to the highest quartile of DHA. After adjustment for age and gender DHA remains a negative predictive factor for insulin resistance. Waist-to-height ratio (WHtR), a marker of visceral obesity was higher in children with a higher AA/(DHA+EPA) ratio. Conclusions: In obese children, the AA is higher in concordance with insulin resistance. Additionally, children with a higher AA/(DHA+EPA) ratio have greater BMI, fat mass, waist circumference, and WHtR, important indicators of central adiposity, and cardio-metabolic disorders. LC/MS is a versatile tool for Omega ratio assessment, especially in children where the sample size is a limiting factor for metabolic and nutrition evaluation.
\end{abstract}

Keywords: omega fatty acids, polyunsaturated fatty acids, obesity, HOMA-IR, insulin resistance Received: $8^{\text {th }}$ November 2020; Accepted: $2^{\text {th }}$ November 2020; Published: $9^{\text {th }}$ December 2020

* Corresponding author: Adina Huțanu, Department of Laboratory Medicine, George Emil Palade University of Medicine, Pharmacy, Science, and Technology of Targu Mures, Romania. E-mail: adina_hutanu03@yahoo.com 


\section{Introduction}

Obesity, both in adults and children is a major public health issue, as this condition seems to become an epidemic during the last years. According to the World Health Organization (WHO), in 2016 more than 650 million adults were obese and over 340 million children and adolescents aged between 5-19 were categorized as obese or overweight (1). Since obesity is a preventable condition, it is important to evaluate its impact on children and adolescents and to identify possible biomarkers as a tool for obese patient evaluation. Aside from genetics, a great impact on obesity is linked with food behavior, and as it is well known, the Western diet is rich in saturated fats and the ratio between polyunsaturated and saturated fatty acids is balanced in favor of the latter, in a ratio of 20:1 in lieu of $1: 1$ (2). In addition to obesity, there are many other interlinked pathological conditions associated with a low polyunsaturated fatty acids (PUFA) intake, like cardiovascular diseases, diabetes, or cancer (3). Obesity implies a low grade of inflammation which is in part modulated by the imbalance between arachidonic acid (AA) an Omega-6 fatty acid, and two Omega-3 fatty acids, docosahexaenoic acid (DHA) and eicosapentaenoic acid (EPA). In addition to the anti-inflammatory effect induced by Omega-3 FAs via specific pro-resolving mediator (SPM), e.g. resolvins derived from EPA and protectins and maresins derived from DHA, the two FAs additionally modulate the adipocyte life cycle (differentiation and apoptosis) (4). Obesity leads to a chronic low-grade inflammatory state, and the therapeutic manipulation of inflammation can be explored. González-Périz and collab. showed in an experimental study in obese mice that Omega-3 supplementation improves the insulin sensitivity in adipose tissue, making a link between inflammation, obesity, and insulin resistance (5). A study performed by Dangardt and col- lab. revealed that Omega-3 supplementation for three months in obese adolescents significantly improved glucose and insulin homeostasis, but only in female subjects (6). A recent analysis of anthropometric parameters in patients with obesity and type 2 diabetes mellitus (DM) in adults provides a comprehensive study regarding the relationship of markers of central adiposity with insulin resistance (7). Omega-3 index inversely correlates to obesity and insulin resistance in children (8) however, the Omega-3 index, representing the proportion of (DHA+EPA) from all FAs (9), imply characterization of the profile of Red Blood Cell (RBC) membranes of all FA (saturated and unsaturated) which could be quite expensive for a routine determination. Despite the fact that a good tool for FAs quantification is the evaluation from the RBC membranes by Liquid Chromatography-Mass Spectrometry (LC/MC) (10), the serum or plasma detection is also a facile way for free fatty acids assessment $(11,12)$. The data from the literature regarding the possible link between DHA, EPA, AA, and total body fat volume, insulin sensitivity, and fat distribution in children and teenagers, are scarce. In this regard, the present study aims to analyze the plasma levels of AA, DHA and EPA, FAs mainly involved in fine tuning of low-grade inflammation, as well the relationship between $\mathrm{AA} /(\mathrm{DHA}+\mathrm{EPA})$ named here Omega Ratio (Omega R) and anthropometric and biochemical parameters in obese and non-obese participating children. Additionally, a potential relationship between plasma FAs and AA/DHA+EPA with markers of insulin resistance (IR) will be discussed. A brief description of LC/MS method validation will be also presented. PUFAs can be quantified using various techniques such as Liquid Chromatography-Mass Spectrometry (LCMS), or Gas Chromatography-Mass Spectrometry (GC-MS) from a wide array of matrices such as plasma, red blood cell membranes and other types of tissue $(10,13-16)$. Both techniques of- 
fer good selectivity and high sensitivity, and are thus suitable for use in PUFAs biomonitoring studies $(13,16,17)$. When selecting a technique to use in PUFAs quantification, it is important to consider not only the selectivity and sensitivity which need to be achieved, but also the robustness of a given technique and/or equipment, and other factors which can affect the validation process for the final method. It is also important to consider the reproducibility of results during each step of the sample preparation and optimize each process in order to obtain high recovery and extraction yields as well as reduced or no contamination (or cross-contamination of samples). In this regard, the Quadrupole Time-of-Flight (QTOF) LC-MS technique offers very high sensitivity and selectivity, but it is necessary to finetune ionization and detection in order to obtain the robustness required for analyzing large numbers of samples (18).

\section{Materials and Methods}

\section{Patient and study design}

This observational study included 213 children aged between 5 to 18 years, who attended the Endocrinology and Pediatric Clinics from Târgu Mureș between 2016-2018. The study protocol received the approval from the Ethical Committee of the University of Medicine and Pharmacy of Târgu Mureș (7/Jan 11 $1^{\text {th }} 2016$ ), and was conducted in accordance with the Helsinki Declaration; before the children's admission in the study according to inclusion/exclusion criteria, all parents had signed the informed consent. Children were divided according to WHO criteria in the overweight or obese group (BMI $>+1$ SD) and normal group, 130 and 83, respectively. The presence of exclusion criteria was defined as follows: all chronic conditions like cardiac, renal, liver insufficiency, any endocrine disease without treatment, malabsorption syndrome, genetic disease, short stature according to the Prad- er growth chart (19), or no signed consent. Of them, ten participants were excluded due to the insufficiency of the sample volume for testing. The subjects were not under Omega fatty acid products supplementation and as the majority of the population, were adepts of the Western diet.

\section{Clinical evaluation, anthropometric data, and blood sample collection}

Clinical evaluation was performed by an experimented endocrinologist using standardized protocols for obesity assessment: height $(\mathrm{cm})$, weight $(\mathrm{kg})$, body mass index (BMI) $\left(\mathrm{kg} / \mathrm{m}^{2}\right)$ expressed as raw values and standard deviation (SD) score, waist circumference (WC), waist-toheight ratio (WHtR) as a marker of central adiposity, tricipital skinfold thickness (TST), and body fat composition using bio-impedance analysis (total fat mass, percentage of fat mass, freefat mass) as previously described (20). For lipids, glucose and endocrine profile (Insulin, Visfatin, RBP-4) the blood was withdrawn after at least 8 hours fasting, during the morning, in EDTA collection tubes. The biochemical parameters were analyzed using routine laboratory procedures, while Insulin (DRG International), Visfatin (Sigma-Aldrich Co.), and RBP-4 (Sigma-Aldrich Co.) were analyzed using enzyme-linked immunosorbent assay (ELISA) according to manufacturers' instructions on automated ELISA platform Dynex DSX (Dynex Technologies). Insulin resistance (IR) and beta-cell function were evaluated using the Homeostasis Assessment Model HOMA-IR [glucose $(\mathrm{mmol} / \mathrm{L}) \mathrm{x}$ insulin $(\mathrm{mIU} /$ $\mathrm{mL})] / 22.5$, and HOMA- $\beta$ [20 $\mathrm{x}$ insulin (mIU/ $\mathrm{mL})] /$ [glucose $(\mathrm{mmol} / \mathrm{L})$ - 3.5] respectively. An Index above 6.0 was consistent with IR, the index below 1.4 was relevant for the absence of IR, while an intermediate group had an index between the above-mentioned values (11). Since obesity is related to altered metabolism of triglycerides and glucose, the triglyceride/glucose 
index (TyG) calculated according to the formula: $\ln [\mathrm{Tg}(\mathrm{mg} / \mathrm{dl})$ x glucose $(\mathrm{mg} / \mathrm{dl}) / 2]$ was also used as a possibility for IR estimation (21-24), a value above 7.88 for children being considered a cut off for insulin resistance assessment risk (24).

\section{Diet assessment}

A food frequency questionnaire (FFQ) comprising 127 items was used, filled in by the legal representative/parent. The methodology for diet assessment was previously described (25); in short, after filling the questionnaire, the application returns the personal food pyramid comprising the 6 food groups - sweets, fat, meat \& proteins, dairy, fruits \& vegetables, and cereal.

\section{Fatty acids measurements (development and full validation method not published yet)}

The method used for the measurement of free AA, EPA, and DHA concentrations from human plasma was developed to be sensitive and selective, but also robust and rapid, allowing for the analysis of a large number of samples in a short period of time. The method was validated according to international guidelines with regard to the most critical parameters (26). Protein precipitation was used for sample preparation, a rapid high-throughput sample preparation method which is also simple and thus minimizes preparation errors and the risk of sample contamination or cross-contamination. An LC-MS system comprised of an AB Sciex (Framingham, USA) QTOF 4600 mass spectrometer coupled with a Perkin-Elmer (Waltham, USA) Flexar FX-10 HPLC system (composed of two single pumps with inline degasser, auto-sampler, and column thermostat) were used for analytical separation and detection. HPLC grade acetonitrile and formic acid $98-100 \%$ of analytical grade were manufactured by Scharlau (Barcelona, Spain). Analytical grade ammonium formate manufactured by Fluka (Buchs, Switzerland) and ultrapure wa- ter (Millipore Direct-Q 3 Water purifier system, Milford, USA) were used. AA, DHA and EPA analytical standards were purchased from ChromaDex (Los Angeles, USA), arachidonic-d11 acid analytical standard was purchased from Cayman Chemical (Ann Arbor, USA).

Before being analyzed using the LC-MS method, plasma samples were precipitated with acetonitrile (ratio of 1:3), at the same time the internal standard (arachidonic-d11 acid) being added. After mixing, samples were centrifuged and the supernatant was transferred to clean HPLC vials in order to be injected into the LC-MS system. The bioanalytical method used for the quantification of AA, DHA, and EPA achieved good analytical separation using a Kinetex XB-C18 analytical column with a mobile phase consisting of acetonitrile and $10 \mathrm{mM}$ ammonium formate aqueous solution in isocratic flow. Ionization of analytes and internal standard was performed using negative electrospray ionization (ESI) ion source. Fragmentation patterns were studied and specific fragments were selected and monitored for the detection of each analyte. The concentration intervals for calibration curves were 2.5-125 $\mu \mathrm{g} / \mathrm{mL}$ for AA and $50-2500 \mathrm{ng} / \mathrm{mL}$ for both DHA and EPA. The method was validated with regards to carryover, selectivity, linearity, between-run and within-run accuracy and precision, and recovery. Short-term, table-top and autosampler stability of solutions were also checked and were found to be satisfactory. In order to assure the validity of results measured from the plasma samples collected from subjects linearity of calibration curves, as well as the accuracy of recalculated calibration standards was monitored during each run, while quality control samples were injected at the end of each analytical sequence in order to verify system accuracy and stability. During all the analytical runs linearity, the accuracy of calibration standard samples proved to be within acceptable limits, and 
the analytical method was shown to be robust and give accurate results (as quality control samples were with acceptable limits for each run) during the whole duration of runs.

\section{Statistical analysis}

The data were analyzed using SPSS v 25.0 (SPSS. Inc, Chicago, USA). Continuous variables were expressed as mean \pm standard deviation (SD) or median and interquartile range, depending on the distribution, and compared using the $t$-test or Mann-Whitney test respectively, normality was tested using the Shapiro-Wilk test. For correlation analysis, the Spearman coefficient was calculated. Categorical variables were expressed as proportions. For testing the association between categorical variables, either Chi-square or Fisher's test was used. The obese group was studied using quartiles, highlighting the difference between first and fourth quartile, for a better visualization of the difference between plasma FFAs analyzed expressed as $\mathrm{AA} / \mathrm{DHA}+\mathrm{EPA}$ ratio. For the FFQ extreme values, $95 \%$ winsorization was used to deal with extreme values. A two-tail p-value $<0.05$ was considered statistically significant.

\section{Results}

\section{Overall characteristics of the study popula- tions}

Plasma levels of free fatty acids were measured using a validated high-throughput LC-MS method in 202 children (127 obese and 75 non-obese), age-, and sex-matched $(\mathrm{p}=0.262)$. No difference was observed in baseline plasma levels of FFAs or Omega ratio between boys and girls (table 1). Briefly, WC was found higher in boys, the percentage of the fat mass (\%) was higher in girls, while WHtR a reliable indicator of central adiposity, was higher in boys at the limit of statistical significance (table 1).

Comparative analysis of plasma free fatty acids profile in obese and non-obese children Since the differences between obese and nonobese groups regarding the demographic and

Table 1. Characteristics of the study population. $y=y e a r s, B M I=b o d y$ mass index, $W C=$ waist circumference, $W H$ tR=waist to height ratio, $A A=$ arachidonic acid, $D H A=$ docosahexaenoic acid, $E P A=$ eicosapentaenoic acid, Omega $R=A A / D H A+E P A, T y G=t r i g l y c e r i d e / g l u c o s e ~ i n d e x, H O M A$ $I R=$ homeostasis model assessment for insulin resistance, $\mathrm{HOMA- \beta =homeostasis} \mathrm{model} \mathrm{assessment}$ for beta-cell function. Values expressed as mean $\pm S D$ or median (interquartile range).

\begin{tabular}{lccc}
\hline Variable & Girls $(\mathbf{n}=\mathbf{1 0 2})$ & Boys $(\mathbf{n}=\mathbf{1 0 0})$ & $\mathbf{p}$ \\
\hline Age $($ Years $)$ & $11.7 \pm 2.9$ & $11.4 \pm 2.9$ & 0.437 \\
\hline Obesity $(\mathrm{n}, \%)$ & $60(47.2 \%)$ & $67(52.7 \%)$ & 0.229 \\
\hline Body Weight $(\mathrm{kg})$ & $47.95(\mathrm{IQR} 39.0-59.4)$ & $53.8(\mathrm{IQR} 39.4-66.0)$ & 0.090 \\
\hline WC $(\mathrm{cm})$ & $77.59 \pm 14.3$ & $82.2 \pm 15.9$ & 0.030 \\
\hline WHtR & $0.52 \pm 0.09$ & $0.55 \pm 0.08$ & 0.051 \\
\hline Fat Mass $(\%)$ & $29.2 \pm 8.8$ & $25.5 \pm 11.4$ & 0.011 \\
\hline HOMA IR & $5.53(\mathrm{IQR} 4.06-7.81)$ & $5.66(\mathrm{IQR} 3.94-8.45)$ & 0.997 \\
\hline HOMA $\beta$ & $119.8(\mathrm{IQR} 80.2-170.1)$ & $118.96(\mathrm{IQR} 87.7-165.8)$ & 0.930 \\
\hline TyG index & $8.03 \pm 0.45$ & $7.94 \pm 0.52$ & 0.179 \\
\hline AA $(\mu \mathrm{g} / \mathrm{mL})$ & $20.66(\mathrm{IQR} 15.45-26.97)$ & $21.54(\mathrm{IQR} 15.75-29.86)$ & 0.495 \\
\hline DHA $(\mathrm{ng} / \mathrm{mL})$ & $370.19(\mathrm{IQR} 283.6-480.9)$ & $373.3(\mathrm{IQR} 297.58-500.5)$ & 0.685 \\
\hline EPA $(\mathrm{ng} / \mathrm{mL})$ & $65.12(\mathrm{IQR} 46.25-92.1)$ & $69.46(\mathrm{IQR} 53.0-97.54)$ & 0.326 \\
\hline Omega R & $49.9 \pm 18.2$ & $50.82 \pm 17.3$ & 0.714 \\
\hline
\end{tabular}


anthropometric parameters were analyzed elsewhere (20), in this subsequent study we performed the analysis of the basal FAs plasma levels in the above mentioned groups (Table 2). There was no significant difference for DHA and EPA between the two groups, however, plasma concentrations of AA, Omega R, TyG and HOMA indices were significantly higher in obese compared to non-obese children ( $\mathrm{p}=0.004$ for $\mathrm{AA}$ and $\mathrm{p}<0.0001$ for the rest) (figure 1 ).

Table 2. Baseline plasma free fatty acids and insulin resistance indices (HOMA, TyG) in non-obese vs obese children AA=arachidonic acid, DHA=docosahexaenoic acid, EPA=eicosapentaenoic acid, Omega R=AA(ng/ $\mathrm{ml}$ )/DHA+EPA, TyG=triglyceride/glucose index, HOMA-IR=homeostasis model assessment for insulin resistance, HOMA- $\beta=$ homeostasis model assessment for beta-cell function. Values expressed as median (interquartile range).

\begin{tabular}{lccc}
\hline Parameter & Non-obese group $(\mathbf{n}=\mathbf{7 5})$ & Obese group $(\mathbf{n}=\mathbf{1 2 7})$ & p value \\
\hline AA $\mu \mathrm{g} / \mathrm{mL}(\mathrm{IQR})$ & $19.44(15.46-24.72)$ & $22.94(15.74-30.50)$ & 0.042 \\
\hline DHA ng/mL $(\mathrm{IQR})$ & $405.78(325.45-481.48)$ & $346.38(265.40-493.55)$ & 0.112 \\
\hline EPA ng/mL $(\mathrm{IQR})$ & $65.11(52.23-80.91)$ & $69.35(47.38-100.35)$ & 0.252 \\
\hline Omega R $(\mathrm{IQR})$ & $42.44(34.82-49.36)$ & $50.39(41.79-64.27)$ & $<0.0001$ \\
\hline TyG $(\mathrm{IQR})$ & $4.20(4.12-4.36)$ & $4.37(4.26-4.55)$ & $<0.0001$ \\
\hline HOMA IR $(\mathrm{IQR})$ & $4.28(3.01-6.01)$ & $6.64(4.83-8.86)$ & $<0.0001$ \\
\hline HOMA- $\beta(\mathrm{IQR})$ & $98.18(65.93-122.04)$ & $138.61(103.64-181.49)$ & $<0.0001$ \\
\hline
\end{tabular}
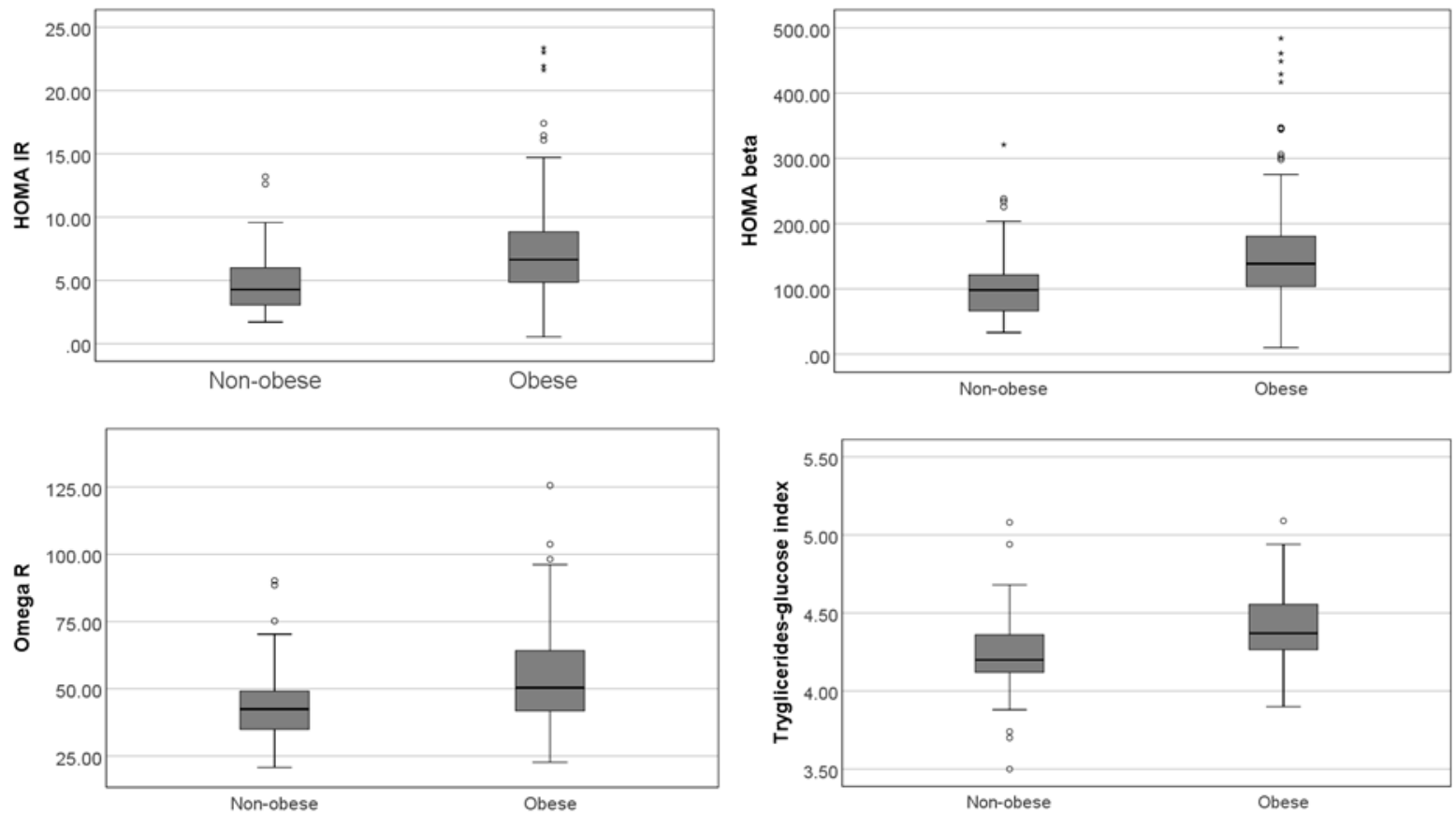

Fig. 1. The parameters (HOMA-IR, Homa- $\beta$ Omega R, TyG index) with statistically significance between non-obese and obese groups. Omega $R$ represents the ratio between $A A$

(Omega-6) and DHA+EPA (Omega-3) FAs 
Association of Omega $R$ with anthropometric parameters, biochemical and hormonal profile Because the AA/DHA+EPA ratio reflects the imbalance between pro- and anti-inflammatory effect of the above-mentioned fatty acids, the whole study group (obese and non-obese) was stratified according to median value of Omega $\mathrm{R}$, a higher value of Omega $\mathrm{R}$ indicating a prevalence of AA to the detriment of Omega-3 FAs. The demographic and baseline laboratory characteristics dichotomized according to the median overall value of Omega $\mathrm{R}$ of 46.13 are detailed in Table 3. In subjects with higher Omega $\mathrm{R}$ and preponderant pro-inflammatory effects exerted by the imbalance between Omega-6 and Omega- 3 in the favor of Omega-6, the BMI_SD, waist_SD, fat mass percent, and WHtR were significantly high- er. Apart from altered anthropometric parameters, the HOMA indices are not statistically different between the two groups, tailored in this fashion. When the same parameters were analyzed for the lowest and highest quartile of plasma EPA and DHA, we found that the anthropometric and most of the plasma parameters showed no difference, whereas plasma insulin levels, HOMA-IR, and HOMA- $\beta$ were significantly higher in the lower quartile group (Q1) of DHA with no differences for EPA interquartiles (Table 4).

After adjustment for age, gender and obese status, levels of EPA $\left(r_{s}=0.219, p=0.002\right)$ and DHA $\left(r_{\mathrm{s}}=0.151, \mathrm{p}=0.033\right)$ positively correlate with the number of fruit $\&$ vegetable portions/day. None of the other food groups influence the fatty acid levels.

Table 3. The difference of studied parameters between lower and higher Omega $\mathrm{R}$ groups. Omega $\mathrm{R}=\mathrm{AA} /$

DHA+EPA, AA=arachidonic acid, DHA=docosahexaenoic acid, EPA=eicosapentaenoic acid, HOMAIR=homeostasis model assessment for insulin resistance, HOMA- $\beta=$ homeostasis model assessment for betacell function. Values expressed as mean \pm SD or median (interquartile range).

\begin{tabular}{lccc}
\hline & $\begin{array}{c}\text { Omega R } \\
\mathbf{4} \mathbf{4 6 . 1 3} \\
(\mathbf{n = 1 0 1})\end{array}$ & $\begin{array}{c}\text { Omega R } \\
\mathbf{4} \mathbf{4 6 . 1 3} \\
\mathbf{( n = 1 0 1 )}\end{array}$ & p value \\
\hline Demographic data & & & \\
Gender Male (n., \%) & $49(48.5 \%)$ & $51(50.5 \%)$ & 0.778 \\
Age (years, mean \pm SD) & $11.4 \pm 2.9$ & $11.8 \pm 2.9$ & 0.927 \\
\hline Anthropometric data & & & \\
BMI_SD & $0.95(-0.13-1.88)$ & $1.57(1.13-2.27)$ & 0.0009 \\
Waist_SD & $1.56(0.31-2.52)$ & $2.01(1.04-3.06)$ & 0.009 \\
WHtR & $0.51(0.45-0.58)$ & $0.55(0.50-0.60)$ & 0.004 \\
Fat_mass_kg & $11.7(6.77-17.97)$ & $15.80(9.82-22.45)$ & 0.006 \\
Fat_mass_(\%) & $24.60(18.80-32.20)$ & $29.80(22.35-36.02)$ & 0.005 \\
Non_fat_mass & $36.00(27.65-41.22)$ & $36.20(26.35-44.95)$ & 0.558 \\
Muscle mass & $34.50(27.52-3.47)$ & $36.20(26.35-44.95)$ & 0.208 \\
\hline Laboratory data & & & \\
AA $\mu$ g/mL & $18.68(14.73-24.32)$ & $24.53(17.73-32.01)$ & $<0.0001$ \\
DHA ng/mL & $425.41(331.01-548.21)$ & $326.24(241.25-437.65)$ & $<0.0001$ \\
EPA ng/mL & $65.33(951.16-96.42)$ & $66.47(47.08-94.21)$ & 0.727 \\
Insulin $(\mu I U / m L)$ & $27.08(19.86-38.97)$ & $29.49(21.92-38.67)$ & 0.354 \\
HOMA-IR & $5.37(3.74-7.69)$ & $5.98(4.41-8.27)$ & 0.302 \\
HOMA- $\beta$ & $112.63(84.93-165.38)$ & $124.39(91.77-170-09)$ & 0.405 \\
TyG & $4.31(4.14-4.45)$ & $4.36(4.19-4.52)$ & 0.231 \\
\hline
\end{tabular}


Table 4. Patients characteristics of anthropometric, biochemical and hormonal profile for the lowest (Q1) and highest (Q4) quartile for plasma DHA. DHA=docosahexaenoic acid, TyG=trygliceride/glucose index, HOMA IR=homeostasis model assessment for insulin resistance, HOMA- $\beta=$ homeostasis model assessment for betacell function. Values expressed as median (interquartile range) or mean and standard deviation (SD).

\begin{tabular}{lccc}
\hline \multirow{2}{*}{ Parameter } & \multicolumn{2}{c}{ DHA Quartile } & p value \\
\cline { 2 - 4 } & $\mathbf{Q 1}(\mathbf{N}=\mathbf{4 1})$ & $\mathbf{Q 4}(\mathbf{N}=\mathbf{4 9})$ & \\
\hline BMI_SD (IQR) & $1.57(1.75-2.12)$ & $1.50(-0.36-2.53)$ & 0.541 \\
\hline Fat mass (\%) & $29.10(22.47-33.72)$ & $27.4(18.7-38.57)$ & 0.981 \\
\hline Muscle mass (median, IQR) & $37.3(27.05-46.25)$ & $34.5(25.12-39.70)$ & 0.09 \\
\hline Waist_SD (median, IQR) & $2.15(1.56-2.60)$ & $1.90(0.54-2.76)$ & 0.303 \\
\hline Glucose mg/dL (mean, SD) & $82.7(7.98)$ & $83.2(8.42)$ & 0.751 \\
\hline Total Cholesterol mg/dL (median, IQR) & $152.0(135.7-175.2)$ & $164.0(150.6-187.9)$ & 0.012 \\
\hline Tryglicerides mg/dL (median, IQR) & $62.0(48.6-96.2)$ & $65.0(49.9-88.4)$ & 0.875 \\
\hline TyG & $4.29(4.16-4.50)$ & $4.31(4.17-4.45)$ & 0.932 \\
\hline HOMA IR (IQR) & $7.27(5.08-10.2)$ & $5.05(3.97-6.91)$ & 0.004 \\
\hline HOMA $\beta(\mathrm{IQR})$ & $129.1(111.7-226.3)$ & $103.9(79.04-159.5)$ & 0.002 \\
\hline Visfatin ng/mL (median, IQR) & $12586(9854-14674)$ & $13225(11588-16106)$ & 0.122 \\
\hline RBP-4 $(\mathrm{ng} / \mathrm{mL})($ median, IQR) & $14436.0(6999.7-22688.5)$ & $10138(6898.4-15750.0)$ & 0.164 \\
\hline Insulin $\mu \mathrm{IU} / \mathrm{mL}($ median, IQR) & $33.75(25.11-52.8)$ & $25.19(19.96-33.83)$ & 0.002 \\
\hline
\end{tabular}

Spearman correlation matrix between studied variables (FAs, HOMA-IR, HOMA- $\beta$, TyG, obesity, insulin)

The Spearman analysis showed a significant correlation between Omega $\mathrm{R}$ and anthropometric data, for BMI_SD $(\mathrm{r}=0.244, \mathrm{p}=0.0001)$, WHtR $(r=0.213, p=0.002)$, fat mass $\%(r=0.224$, $\mathrm{p}=0.002$ ). A significant negative correlation was maintained between DHA and HOMA-IR ( $\mathrm{r}=-$ $0.204, p=0.004$ ), while the correlation between EPA or Omega Ratio and HOMA-IR was lost after age and gender adjustment.

\section{Discussion}

In our study, we sought to investigate the difference of three important PUFAs (DHA and EPA along with $\mathrm{AA}$ ) in a relatively large sample size of obese and non-obese children and to evaluate the potential link between the above mentioned FFAs measured in plasma and fat percentage, muscle mass, and insulin resistance scores. Although the quantification of FAs in the RBC membranes is desirable, plasma FAs detection can also provide valuable information. As in Burrows et al (8), we did not find a statistical difference for Omega-3 FFAs (DHA and EPA) between obese and non-obese groups, however, AA a potent pro-inflammatory fatty acid and AA/DHA+EPA (defined as Omega R) were significantly higher in obese children $(p=0.042$ for AA and $p<0.0001$ for Omega $R$ ), highlighting in this way the imbalance between pro-inflammatory and anti-inflammatory effects of FAs in obesity. The unbalance between AA and DHA+EPA will determine the maintenance of a pro-inflammatory status and develop an insulin resistance, highlighted by HOMA-IR $>6$ and higher insulin plasma levels, more prevalent in patients with low DHA levels. DHA and EPA could be synthesized from alfa linolenic acid (ALA) or brought into the body through diet. Because the conversion from ALA is not very efficient, DHA and EPA are considered essential nutrients and, therefore, they must be supplemented by diet or food supplements. While the mediterranean diet is rich in Omega 3 FAs, the Western diet which prevails in our region is an important obesity in- 
ducing factor (27) having an Omega-6/Omega-3 ratio of 20:1 (2), very rich in pro-inflammatory FAs.

The adipose tissue could be considered as an organ with metabolic and endocrine activity, many cytokines and hormones with autocrine and paracrine action being secreted by adipocytes (28). During childhood, the adipose tissue has a great capacity to adapt in relation to many external factors, an important one being the recruitment of new adipocytes, increasing the adipocyte size, increasing the white adipose mass, with consecutive alteration in adipokine secretion (29). As expected, the excessive adipose tissue exerts negative metabolic actions associated with insulin resistance, dyslipidemia, and diabetes, as well as with prothrombotic state and low-grade inflammation (28). This inflammatory state driven by the adipose tissue is, among others, the consequence of obesity-induced M2 anti-inflammatory macrophages to pro-inflammatory M1 macrophages shift (30). Some experimental data suggest a link between PUFAs and adipocyte function, modulated throughout the increasing the beta-oxidation of FAs and inhibiting hepatic lipogenesis with consecutive total body fat reduction. Extrapolating, these effects could improve insulin sensitivity (30). However, in humans with already set type 2 diabetes mellitus (DM), insulin sensitivity failed to improve after Omega-3 supplementation, the inadequate control or dosage being suspected (30). Omega 6/Omega 3 ratio exerts influences upon adipogenesis and lipid metabolism, inflammation, and brain-gut-adipose tissue. The last ones are mediated throughout the endocannabinoid system, being responsible for activation of $\mathrm{CB} 1$ and $\mathrm{CB} 2$ receptors, and upregulated by increased concentration of AA (2). The mediterranean diet has a great impact on the prevention of obesity and conditions related to obesity diseases; however, because of the low adherence to mediterranean diet, obesity becomes endemic among the youngsters in European countries (27), but not only. This aspect is of particular interest in children; the low adherence to mediterranean diet, higher intake of processed food, rich in trans fatty acids, and low physical activity being unhealthy habits conveying to obesity. Thus, the main difference between Western and Mediterranean diets consists mainly of fatty acids composition. While the mediterranean diet is rich in monounsaturated fatty acids (MUFAs) preponderantly found in olive oil and polyunsaturated fatty acids (PUFAs) mainly found in marine products, the Western diet is rich in saturated fatty acids (SFAs) (3). In the last years, the increasing prevalence of type $2 \mathrm{DM}$ in children is obviously increased, this pathology is no longer a disease of adult age. In addition to hereditary, polygenic susceptibility in the pathogenesis of type $2 \mathrm{DM}$, the environmental, lifestyle, and nutritional factors being substantial contributors. Genetic factors have strong interlinks and in addition to associated factors are eventually responsible for obesity promotion. There are many epigenetic modifications that might be studied in relation to obesity and related complications; among them, microRNAs (miRNA) derived from adipocytes involved in TGF- $\beta$ and $\mathrm{Wnt} / \beta$ catenin pathways, which seems to be altered in childhood obesity, is a promising tool (31). A recent publication endorses the importance of exosomes in the pathogenesis of obesity and insulin resistance, adipocyte-derived exosomes playing a crucial role in macrophage activation and proinflammatory response, although there are exosomes that carry out information that blunts the inflammation, throughout the M2 macrophage polarization (32). On the other hand, the crosstalk between $\beta$-cells and insulin-sensitive organs for glycemic homeostatic purpose could imply exosomes, the number of circulating extracellular vesicle correlates with HOMA- $\beta$ (32). Despite the small size of the extracellular vesicle (up to $1000 \mathrm{~nm}$ ), exosomes seem to be a prom- 
ising marker not only in obesity-related disease but also in malignant disease, since they transmit the information from descent cells to target tissues (31,33-35). There is a strong relationship between adipose and muscular tissues and the glucose homeostasis, throughout the regulation of glucose uptake from the periphery via two high-affinity transporters, one insulin-dependent GLUT4, and the other one insulin-independent GLUT1 in a ratio of 3.5:1 $(36,37)$. In both aforementioned tissues, insulin increases the membrane expression of the GLUT4 from the cytoplasmic vesicle, facilitating the glucose uptake in postprandial state. In insulin-resistant subjects the decreased rate of glucose uptake has different characteristics in adipose compared to muscular tissue. A study using Positron Emission Tomography (PET) for the measurement of blood supply and glucose uptake in skeletal and adipose tissue revealed that in adipose tissue the glucose uptake mediated by insulin is impaired due to a reduction in blood flow, while in skeletal muscular tissue in the resting phase, the glucose-insulin homeostasis impairment seems to be a consequence of a cellular defect (36). By decreasing the blood supply due to adipocytes expansion in adipose tissue, a consecutive macrophage infiltration will secrete pro-inflammatory cytokines which in turn will change the profile of adipokine secretion (4). Insulin resistance reflected by impaired glucose uptake in the main insulin-sensitive tissues like muscular and adipose have a different pattern than liver metabolism and is in close relationship with endogenous glucose production (EGP) (38). A strong negative correlation was found between obesity and tissue glucose uptake; besides the antilipolytic effect of insulin in adipose tissue, it affects the glucose uptake by the skeletal muscle tissue and liver glucose production, increasing the imbalance of glucose metabolism (38). A profile of FAs was analyzed in a group of children with newly diagnosed diabetes, onset diabetes and controls by Castro-Correia et al (39); the results revealed that plasma levels of AA in the diabetes was higher than in healthy children, while DHA and EPA levels were significantly lower in controls vs diabetes group, these controversial results probably are due to the very low number of the recruited participants.

In a small size study Burrows et al has studied the relationship between Omega 3 index (defined as the percentage of DHA+EPA from all measured free fatty acids in the erythrocyte membranes) weight and IR in obese and non-obese children (8). The researchers found that the Omega-3 Index is altered in obese children and inversely correlated to insulin levels and HOMA score. An extended Omega-3 index takes into account all FFAs, saturated, monounsaturated, and polyunsaturated fatty acids, however, these are non-routine, quite expensive parameters. Both Omega-3 FAs, DHA, and EPA modulate the adipocyte proliferation, differentiation, and adipokine secretion (4) and are the main players in the fine tuning of low-grade inflammation related to obesity (4). Additionally, PUFA supplementation seems to influence the miRNA synthesis profiles and modulate inflammation by means of cytokine synthesis or other macrophage properties (40).

In a previous study investigating the lipid profile in ischemic stroke patients, we found that in the Romanian population with no obvious illness (control group), the Omega-3 index was very low (41) compared to the recommendations (42). As previous studies revealed that the triglyceride glucose index seems to be a good predictor for insulin resistance $(21,22)$, when analyzed in our study group this parameter found a significant higher TyG index in obese compared to non-obese children $(p<0.0001)$ yet, without a clear difference between the lowest and the highest quartile of Omega $\mathrm{R}$ into the obese group. A recent meta-analysis revealed the effectiveness of Omega 3 supplementation for lowering tri- 
glyceride levels and waist circumference without important influence on BMI or total cholesterol, LDL cholesterol or glucose (43). Because there was a significant relation between WHtR, a marker of central adiposity, and Omega R, with a higher ratio with clear imbalance of Omega 6 and Omega 3 in favor of pro-inflammatory Omega 6, results that are in line with other data from the literature (44), we could suggest that an improvement in AA/DHA+EPA equilibrium by any means of dietary supplementation (fish oil, supplements), may reduce the central adiposity which in turn will improve the insulin sensibility, as we found a higher insulin plasma level in the group with a higher AA profile.

\section{Conclusions}

In obese children, the plasma level of AA is higher in concordance with insulin resistance. Additionally, children with a higher AA/DHA+EPA ratio had greater BMI, fat mass, waist circumference, and waist-to-height ratio, important indicators of central adiposity, and cardio-metabolic disorders. The results of the study subscribe to a small number of studies on the level of FAs in children and the relationship of the Omega Ratio with anthropometric indices, obesity, and insulin resistance. Additionally, LC/MS is a versatile tool for Omega ratio assessment, especially in children where the sample size is a limiting factor for metabolic and nutrition evaluation.

$\begin{array}{ll}\text { Abbreviations } \\ \text { AA } & \text { arachidonic acid } \\ \text { ALA } & \text { alfa linolenic acid } \\ \text { BMI } & \text { body mass index } \\ \text { DHA } & \text { docosahexaenoic acid } \\ \text { EDTA } & \text { Ethylene-diamine-tetraacetic acid } \\ \text { ELISA } & \text { enzyme-linked immunosorbent } \\ \text { EPA } & \begin{array}{l}\text { assay } \\ \text { eicosapentaenoic acid }\end{array}\end{array}$

FA fatty acid

FFA free fatty acid

GC-MS Gas Chromatography-Mass Spectrometry

HOMA Homeostasis Assessment model

HPLC High Performance Liquid Chromatography

IR insulin resistance

LC/MS Liquid Chromatography-Mass Spectrometry

In Natural logarithm

MUFA monounsaturated fatty acids

Omega6/ AA/(DHA+EPA)

Omega3

Omega R AA/(DHA+EPA)

PUFA polyunsaturated fatty acids

RBC red blood cell

RBP-4 Retinol-binding protein 4

SD standard deviation

SFA Saturated fatty acids

SPM specific pro-resolving mediators

TST tricipital skinfold thickness

TyG triglyceride/glucose index

WHtR waist-to-height ratio

WHO World Health Organization

\section{Acknowledgments}

This research was partially supported by an internal research grant from the George Emil Palade University of Medicine, Pharmacy, Science, and Technology of Targu Mures, Romania Grant No.17802/1/22.12.2015, and by the CNFIS grant No. FDI -2018-0567.

\section{Authors' contribution}

AH: designed the study, performed ELISA analysis, analyzed data, wrote the manuscript; MZ performed LC/MC analysis; LF performed $\mathrm{LC} / \mathrm{MC}$ analysis, method validation, wrote the 
manuscript; IMP designed the study provided resources, clinical data and samples, evaluated the manuscript; RP performed the statistical analysis, clinical evaluation; MD resources, interpretation of data, critically evaluated the manuscript, final approval.

\section{Conflict of interest}

The authors declare no conflict of interest.

\section{References}

1. https://www.who.int/news-room/fact-sheets/detail/obesity-and-overweight.World Health Organization. 2020 accessed 01 July 2020.

2. Simopoulos A. An Increase in the Omega-6/Omega-3 Fatty Acid Ratio Increases the Risk for Obesity. Nutrients. 2016;8(3):128. DOI: 10.3390/nu8030128

3. Romagnolo DF, Selmin OI. Mediterranean Diet and Prevention of Chronic Diseases. Nutr Today.2017;52(5):208-22. DOI: 10.1097/ NT.0000000000000228

4. De Mello AH, Uberti MF, De Farias BX, De Souza NAR, Rezin GT. N-3 PUFA and obesity: From peripheral tissues to the central nervous system. Br J Nutr. 2018;119(11):1312-13. DOI: 10.1017/ S0007114518000429

5. González-Périz A, Horrillo R, Ferré N, Gronert K, Dong B, Morán-Salvador E, et al. Obesity-induced insulin resistance and hepatic steatosis are alleviated by $\omega-3$ fatty acids: a role for resolvins and protectins. FASEB J. 2009;23(6):1946-57. DOI: 10.1096/fj.08125674

6. Dangardt F, Chen Y, Gronowitz E, Dahlgren J, Friberg P, Strandvik B, et al. High Physiological Omega-3 Fatty Acid Supplementation Affects Muscle Fatty Acid Composition and Glucose and Insulin Homeostasis in Obese Adolescents. J Nutr Metab. 2012; article ID 395757. DOI: $10.1155 / 2012 / 395757$

7. Cernea S, Both E, Fodor A. The association of anthropometric parameters with markers of insulin and leptin secretion and resistance in type 2 diabetes mellitus. Rev Rom Med Lab. 2020;28(3):299-314. DOI: 10.2478/ rrlm-2020-0028

8. Burrows T, Collins CE, Garg ML. Omega-3 index, obesity and insulin resistance in children. Int J Pediatr Obes. 2011;6(2-2):e532-9. DOI: $10.3109 / 17477166.2010 .549489$

9. Harris WS, Del Gobbo L, Tintle NL. The Omega-3 Index and relative risk for coronary heart disease mortality: Estimation from 10 cohort studies. Atherosclerosis. 2017;262:51-4. DOI: 10.1016/j.atherosclerosis.2017.05.007

10. Tero-Vescan A, Vancea S, Huţanu A, Borka-Balás R, Dobreanu M. Concordance and controversy in determining the omega-3 index in plasma and red blood cells membrane. Farmacia. 2015;63(4):504-9.

11. Meza KS, Pérez CET, Ramírez CAS, Valencia RM, Equihua MDT. Niveles de ácido eicosapentaenoico en escolares obesos con y sin resistencia a la insulina. Nutr Hosp. 2015;31(3):1102-8.

12. Inoue K, Kishida K, Hirata A, Funahashi T, Shimomura I. Low serum eicosapentaenoic acid / arachidonic acid ratio in male subjects with visceral obesity. Nutr Metab. 2013;10(1):25. DOI: 10.1186/1743-7075-10-25

13. Volpato M, Spencer JA, Race AD, Munarini A, Belluzzi A, Cockbain AJ, et al. A liquid chromatography-tandem mass spectrometry method to measure fatty acids in biological samples. J Chromatogr B Anal Technol Biomed Life Sci. 2017;1055-1056:125-34. DOI: 10.1016/j.jchromb.2017.04.030

14. Dillon GP, Keegan JD, Wallace G, Yiannikouris A, Moran CA. The validation \& verification of an LC/MS method for the determination of total docosahexaenoic acid concentrations in canine blood serum. Regul Toxicol Pharmacol. 2018;95:198-203. DOI: 10.1016/j. yrtph.2018.03.021

15. Aslan M, Özcan F, Aslan I, Yücel G. LC-MS/MS analysis of plasma polyunsaturated fatty acids in type 2 diabetic patients after insulin analog initiation therapy. Lipids Health Dis. 2018;12(1):169. DOI: 10.1186/1476511X-12-169

16. Serafim V, Tiugan DA, Andreescu N, Mihailescu A, Paul C, Velea I, et al. Development and validation of a LC-MS/MS-based assay for quantification of free and total omega 3 and 6 fatty acids from human plasma. Molecules. 2019;24(2):360. DOI: 10.3390/molecules 24020360

17. Salm P, Taylor PJ, Kostner K. Simultaneous quantification of total eicosapentaenoic acid, docosahexaenoic acid and arachidonic acid in plasma by high-performance liquid chromatography-tandem mass spectrometry. Biomed Chromatogr. 2011;25(6):652-9. DOI: 10.1002/bmc. 1496

18. Rochat B. Quantitative and Qualitative LC-High-Resolution MS: The Technological and Biological Reasons for a Shift of Paradigm. Recent Advances in Analytical Chemistry. IntechOpen; 2018. DOI: 10.5772/intechopen. 81285

19. Prader A, Largo RH, Molinari L, Issler C. Physical growth of Swiss children from birth to 20 years of age. First Zurich longitudinal study of growth and develop- 
ment. Helv Paediatr Acta Suppl. 1989;52:1-125.

20. Vasilache SL, Mărginean CO, Boaghi A, Pop R, Banescu C, Moldovan VG, et al. Implications of visfatin genetic variants in the metabolic profile of the Romanian pediatric population. Rev Romana Med Lab. 2020;28(2):163-74. DOI: 10.2478/rrlm-2020-0015

21. Lim J, Kim J, Koo SH, Kwon GC. Comparison of triglyceride glucose index, and related parameters to predict insulin resistance in Korean adults: An analysis of the 2007-2010 Korean national health and nutrition examination survey. PLoS One. 2019;14(3):e0212963. DOI: 10.1371/journal.pone.0212963

22. Kim B, Choi HY, Kim W, Ahn C, Lee J, Kim JG, et al. The cut-off values of surrogate measures for insulin resistance in the Korean population according to the Korean Genome and Epidemiology Study (KOGES). PLoS One. 2018;13(11):e0206994. DOI: 10.1371/journal.pone.0206994

23. Simental-Mendía LE, Rodríguez-Morán M, Guerrero-Romero F. The product of fasting glucose and triglycerides as surrogate for identifying insulin resistance in apparently healthy subjects. Metab Syndr Relat Disord. 2008;6(4):299-304. DOI: 10.1089/met.2008.0034

24. Vieira-Ribeiro SA, Fonseca PCA, Andreoli CS, Ribeiro AQ, Hermsdorff HHM, Pereira PF, et al. The TyG index cutoff point and its association with body adiposity and lifestyle in children. J Pediatr.2019;95(2):217-23. DOI: 10.1016/j.jped.2017.12.012

25. Pop RM, Pop M, Dogaru G, Bacarea VC. A web-based nutritional assessment tool. Stud Informatics Control. 2013;22(2):307-14. DOI: 10.24846/v22i3y201307

26. Medicines Agency E. 2** Committee for Medicinal Products for Human Use (CHMP) Guideline on bioanalytical method validation. 2011. www.ema.europa.eu/ contact.

27. D'innocenzo S, Biagi C, Lanari M. Obesity and the mediterranean diet: A review of evidence of the role and sustainability of the mediterranean diet. Nutrients. 2019;11(6):1306. DOI: 10.3390/nu11061306

28. Kershaw EE, Flier JS. Adipose Tissue as an Endocrine Organ. The Journal of Clinical Endocrinology \& Metabolism. 2004;89(6):2548-56. DOI: 10.1210/jc.20040395

29. Rupérez FJ, Martos-Moreno GÁ, Chamoso-Sánchez D, Barbas C, Argente J. Insulin Resistance in Obese Children: What Can Metabolomics and Adipokine Modelling Contribute? Nutrients. 2020;12(11):3310. DOI: 10.3390/nu12113310

30. Kalupahana NS, Claycombe KJ, Moustaid-Moussa N. (n-3) Fatty Acids Alleviate Adipose Tissue Inflammation and Insulin Resistance: Mechanistic Insights. Ad- vances in Nutrition 2011;2(4):304-316. DOI: 10.3945/ an. 111.000505

31. Ferrante SC, Nadler EP, Pillai DK, Hubal MJ, Wang Z, Wang JM, et al. Adipocyte-derived exosomal miRNAs: a novel mechanism for obesity-related disease. Pediatr Res. 2015;77(3):447-54. DOI: 10.1038/pr.2014.202

32. Kim A, Shah A, Nakamura T. Extracellular Vesicles: A Potential Novel Regulator of Obesity and Its Associated Complications. Children. 2018;5(11):152. DOI: 10.3390/children5110152

33. Kanninen KM, Bister N, Koistinaho J, Malm T. Exosomes as new diagnostic tools in CNS diseases. Biochim Biophys Acta - Mol Basis Dis. 2016;1862(3):403410. DOI: 10.1016/j.bbadis.2015.09.020

34. LeBleu VS, Kalluri R. Exosomes as a Multicomponent Biomarker Platform in Cancer. Trends in Cancer. Cell Press 2020;6(9):767-774 DOI: 10.1016/j.trecan.2020.03.007

35. Bălașa A, Șerban G, Chinezu R, Hurghiș C, Tămaș F, Manu D. The involvement of exosomes in glioblastoma development, diagnosis, prognosis, and treatment. Vol. 10, Brain Sciences. 2020;10(8):1-16. DOI: 10.3390/ brainsci10080553

36. Ferrannini E, Iozzo P, Virtanen KA, Honka MJ, Bucci M, Nuutila P. Adipose tissue and skeletal muscle insulin-mediated glucose uptake in insulin resistance: Role of blood flow and diabetes. Am J Clin Nutr. 2018;108(4):749-58. DOI: 10.1093/ajen/nqy162

37. Bouché C, Serdy S, Kahn CR, Goldfine AB. The cellular fate of glucose and its relevance in type 2 diabetes. Endocrine Reviews 2004;25(5):807-30. DOI: 10.1210/ er.2003-0026

38. Honka MJ, Latva-Rasku A, Bucci M, Virtanen KA, Hannukainen JC, Kalliokoski KK, et al. Insulin-stimulated glucose uptake in skeletal muscle, adipose tissue and liver: A positron emission tomography study. Eur J Endocrinol. 2018;178(5):523-31. DOI: 10.1530/EJE17-0882

39. Castro-Correia C, Sousa S, Norberto S, Matos C, Domingues VF, Fontoura M, et al. The Fatty Acid Profile in Patients with Newly Diagnosed Diabetes: Why It Could Be Unsuspected. Hindawi. Int J Pediatr. 2017; article ID 64241861. DOI: 10.1155/2017/6424186

40. Roessler C, Kuhlmann K, Hellwing C, Leimert A, Schumann J. Impact of polyunsaturated fatty acids on miRNA profiles of monocytes/macrophages and endothelial cells-a pilot study. Int J Mol Sci. 2017;18:284. DOI: $10.3390 /$ ijms 18020284

41. Hutanu A, Iancu M, Dobreanu M, Oprea O, Barbu S, Maier S, et al. Extended lipid profile in Romanian ischemic stroke patients in relation to stroke severity and outcome: a path analysis model. Arch Med Sci. 2019. 
DOI: $10.5114 /$ aoms.2019.89302

42. Harris WS. The omega-3 index as a risk factor for coronary heart disease. American Journal of Clinical Nutrition. Am J Clin Nutr. 2008 Jun;87(6):1997S-2002S. DOI: $10.1093 / \mathrm{ajcn} / 87.6 .1997 \mathrm{~S}$

43. Zhang YY, Liu W, Zhao TY, Tian HM. Efficacy of omega-3 polyunsaturated fatty acids supplementation in managing overweight and obesity: A meta-analysis of randomized clinical trials. J Nutr Heal Aging. 2017;21(2):187-92. DOI: 10.1007/s12603-016-0755-5

44. Micallef M, Munro I, Phang M, Garg M. Plasma n-3 polyunsaturated fatty acids are negatively associated with obesity. Br J Nutr. 2009;102(9):1370-4. DOI: $10.1017 / \mathrm{S} 0007114509382173$ 